\title{
Reproducción del pez erizo, Diodon holocanthus (Pisces: Diodontidae) en la plataforma continental del Pacífico Central Mexicano
}

\author{
Gabriela Lucano-Ramírez, Edith Peña-Pérez, Salvador Ruiz-Ramírez, Jorge Rojo-Vázquez \& \\ Gaspar González-Sansón \\ Departamento de Estudios para el Desarrollo Sustentable de Zonas Costeras, Universidad de Guadalajara. V. Gómez \\ Farias 82, San Patricio-Melaque, Jalisco, México. 48980, Jalisco, México. Tel: (052-315) 3556330, Fax (052 315) \\ 3556331; lucanorg@costera.melaque.udg.mx, sruizram@costera.melaque.udg.mx, jrojo@costera.melaque.udg.mx, \\ gaspargonzalez2001@yahoo.com
}

Recibido 03-II-2010. Corregido 12-X-2010. Aceptado 14-XI-2010.

\begin{abstract}
Reproduction of the Spiny Puffer, Diodon holocanthus (Pisces: Diodontidae) in the continental shelf of Mexican Central Pacific. Diodon holocanthus is an important economic and ecological species of the demersal fish community, caught as bycatch from local shrimp fishery. The reproductive biology of this longspine porcupinefish has not yet been described, and reproductive season, the sex ratio, length distribution, length at first gonad maturity, and the gonad macro and microscopic features are described. A total of 400 organisms, ranging from 5.0 to $40.3 \mathrm{~cm}$ (average $18.4 \mathrm{~cm}$ ) total length, were caught from the continental shelf of the Central Mexican Pacific, from December 1995 and December 1998. Sex ratio was 1:0.86 females to males $(\mathrm{n}=253)$. The length at which $50 \%$ of the individuals showed maturing gonads was $19.7 \mathrm{~cm}$ for females and $20.1 \mathrm{~cm}$ for males. Length of the smallest organism with ripe gonads was $12.2 \mathrm{~cm}$ for females and $13 \mathrm{~cm}$ for males. Four gonadal maturation stages were found in both sexes, and five oocyte development phases were identified. The oocyte development pattern is of asynchronous type, which means the species can reproduce several times a year. Testicle development is lobular type, as in most teleost fishes. Monthly mean values of the gonad-somatic index suggest the reproduction activity peaks in June, and September-December. Rev. Biol. Trop. 59 (1): 217 232. Epub 2011 March 01.
\end{abstract}

Key words: reproduction, Diodontidae, balloonfish, gonadal maturation, sex ratio.

La familia Diodontidae consta de dos géneros y 15 especies que se caracterizan por ser peces de talla pequeña a moderada (12 a $30 \mathrm{~cm})$, cuerpo robusto, cabeza grande y obtusa, mandíbulas transformadas en un pico constituido por un sólo diente en cada mandíbula, aberturas branquiales sin opérculo, piel sin escamas típicas y el cuerpo cubierto de espinas muy fuertes (Allen \& Robetson 1994, Bussing \& López 1994). Los peces erizo habitan mares tropicales y templados, son comunes en aguas costeras someras y en ocasiones penetran en aguas salobres y dulces. Generalmente, son solitarios o forman pequeños grupos más o menos dispersos (Bussing \& López 1994). Estas especies prefieren sitios planos abundantes en vegetación, arrecifes coralinos y áreas de mangle (Randall 1967, Nagelkerken \& Dorenhbosch 2000, Patton 2009). Se conoce que Diodon holocanthus Linnaeus 1758, al igual que otros peces e invertebrados marinos, puede contener una sustancia neurotóxica de origen bacteriano en la piel y órganos denominada tetrodotoxina (Chen \& Chou 1998).

Las especies de la familia Diodontidae aparecen en listados ictiofaunísticos del Pacífico mexicano en donde su abundancia se reporta como baja o escasa (Ramírez-Hernández \& 
Carrillo-González 1961, Ramírez-Hernández \& Páez-Barrera 1964, Van Der Heiden \& Findley 1988, Acal \& Arias 1990, Galván-Magaña et al. 1996). En la plataforma continental de los estados de Jalisco y Colima, en México, se han realizado algunos estudios de carácter general sobre los recursos de fondos blandos y rocosos, en donde la familia Diodontidae, está representada con tres especies: Chilomycterus reticulatus (Linneaus 1758), D. hystrix Linnaeus 1758 y D. holocanthus (Aguilar-Palomino et al. 1996, González-Sansón et al. 1997, AguilarPalomino et al. 2001, Lucano-Ramírez et al. 2001a, Rojo-Vázquez et al. 2001). RaymundoHuizar \& Chiapa-Carrara (2000) realizaron un estudio sobre la alimentación de D. histrix y D. holocanthus, y concluyeron que las dos especies presentan un espectro trófico compuesto por 79 y 61 entidades alimenticias, respectivamente, entre los cuales abundan los bivalvos, gasterópodos y braquiuros. Fujita et al. (1997) describieron las características histológicas de los ovarios y el ciclo de reproducción anual de D. holocanthus, en Okinawa, Japón.

Muchas de las investigaciones ictiológicas se enfocan el estudio de los recursos pesqueros de importancia comercial y no se está dando suficiente atención a especies que se capturan, pero que su destino no es el consumo directo, y que pueden ser aprovechadas con menor intensidad debido a la facilidad de captura; sucede lo mismo, con las especies que no poseen valor económico, sin embargo tienen una función ecológica importante en los ecosistemas costeros (Martínez 1988).

A pesar de que $D$. holocanthus se captura de manera regular en la pesca de arrastre no hay estudios biológicos sobre esta especie. El presente trabajo tiene como objetivo principal el analizar los principales aspectos reproductivos y poblacionales de la especie Diodon holocanthus que es capturado en la plataforma continental del Pacífico central mexicano.

\section{MATERIALES Y MÉTODOS}

Área de estudio. La zona de estudio incluye desde Punta Farallón en el estado de Jalisco $\left(19^{\circ} 21^{\prime} \mathrm{N}, 105^{\circ} 01^{\prime} \mathrm{W}\right)$ hasta Cuyutlán en el estado de Colima (18 $\left.{ }^{\circ} 55^{\prime} \mathrm{N}, 104^{\circ} 07^{\prime} \mathrm{W}\right)$. La línea de la costa es irregular y presenta tres accidentes importantes, constituidos por Bahía de Tenacatita, Bahía de Navidad y el grupo de bahías Manzanillo-Santiago, el resto de la línea costera está formada por zonas arenosas relativamente extensas y poco protegidas en donde se localizan las playas Cuitzmala, El Coco, Tepalcates y Cuyutlán (González-Sansón et al. 1997).

Recolecta de muestras. Mensualmente, de diciembre de 1995 a diciembre de 1998, se llevaron a cabo muestreos nocturnos a bordo del Barco de Investigación Pesquera V (BIP V), propiedad de la Universidad de Guadalajara. El BIP V tiene $12 \mathrm{~m}$ de eslora y está equipado para la pesca de arrastre de camarón con redes por ambas bandas. Los arrastres se realizaron con redes camaroneras tipo semiportuguesa, abertura de trabajo en la boca de $6.9 \mathrm{~m}$, altura de relinga de $1.15 \mathrm{~m}$ y tamaño de malla estirada en el copo de $38 \mathrm{~mm}$. La duración promedio de cada arrastre fue de 30 minutos, a una velocidad media de dos nudos.

Los individuos de D. holocanthus fueron separados del resto de la fauna capturada y para su ubicación taxonómica se siguió la descripción de Allen \& Robertson (1994). De cada individuo se registró la longitud total (LT) en centímetros $( \pm 0.1 \mathrm{~cm})$ y el peso total $(\mathrm{PT})$ en gramos $( \pm 0.1 \mathrm{~g})$. Los ejemplares se disectaron para extraer las gónadas y se obtuvo el peso de las mismas $(\mathrm{PG})$ en gramos $( \pm 0.1 \mathrm{~g})$, y el peso de los organismos sin gónada (Psg) en gramos $( \pm 0.1 \mathrm{~g})$. Se registró el sexo y se estimó el estadio de maduración de la gónada mediante la observación macroscópica, tomando como referencia la clasificación cualitativa de Holden \& Raitt (1975) modificada por Santos-Martínez (1989). Las gónadas fueron etiquetadas y preservadas en formol neutro al $10 \%$ para su posterior procesamiento histológico.

El procesamiento histológico consistió en la deshidratación con alcohol etílico en concentración creciente, inclusión en parafina, obtención de cortes de $6 \mu \mathrm{m}$ y tinción con 
hematoxilina-eosina. Se realizaron observaciones con un microscopio de luz Axiostar Plus (Zeiss). Para la descripción microscópica del ovario se tomaron como referencia los trabajos realizados por Yamamoto \& Yamazaki (1961), Cerisola (1984), Peralta-Carranco (1991) y Lucano-Ramírez et al. (2001c). Para la descripción microscópica del testículo se utilizaron los trabajos realizados por Hyder (1969) y LucanoRamírez (1998). Se obtuvieron mediciones de los ovocitos con una Cámara Digital Motic acoplada al microscopio y conectada a una computadora.

Se calculó el índice gonadosomático (IGS) de cada individuo mediante la expresión propuesta por Rodríguez-Gutiérrez (1992):

$$
\mathrm{IGS}=(\mathrm{PG} / \mathrm{Psg}) * 100
$$

donde: PG es el peso de la gónada, Psg es el peso del pez sin gónada

Se analizó la proporción sexual considerando la muestra total, anual y por clase de longitud. Las probables diferencias entre sexos se analizaron mediante la prueba de $\mathrm{Ji}^{2}$ (Zar 1996).

Para estimar la longitud total en donde el $50 \%$ de los individuos se encontraban maduros sexualmente $\left(L_{50}\right)$, se ajustó la ecuación logística a la fracción de peces maduros que presentaron gónadas en los estadios de desarrollo II, III y IV de acuerdo a la escala de Holden \& Raitt (1975) modificada por Santos-Martínez (1989), y se procedió según la expresión

$$
\mathrm{P}_{L}=1 /\left[1+\mathrm{e}^{(a x+b)}\right]
$$

donde: $\mathrm{P}_{L}$ es el porcentaje de individuos maduros a la longitud x, $a$ y $b$ son parámetros de la ecuación de regresión (Echeverría 1987).

Mediante el programa STATGRAPHICS 7.0 se realizaron diferentes análisis de varianza (ANDEVA), en donde se comparó el diámetro de los ovocitos para los distintos meses y fases de desarrollo, la longitud promedio por sexo y las variaciones del IGS promedio mensual de hembras y machos. Cuando se rechazó la hipótesis nula del ANDEVA, se empleó la prueba de comparaciones múltiples de Tukey (Zar 1996). Todas las pruebas se realizaron con nivel de significación de 0.05 .

\section{RESULTADOS}

Talla y composición por sexo. Se recolectaron en total 400 individuos de $D$. holocanthus, los cuales presentaron una amplitud de tallas entre 5.0 y $40.4 \mathrm{~cm}$ y la talla promedio fue de $18.5 \pm 0.35 \mathrm{~cm}$; la mayor frecuencia de individuos se encontró en la marca de clase de $11.0 \mathrm{~cm}$. Los organismos a los cuales no se les identificó el sexo presentaron longitud promedio de $12.9 \pm 0.39 \mathrm{~cm}$ (longitud mínima y máxima de $5.0 \mathrm{~cm}$ y $29.0 \mathrm{~cm}$ respectivamente) Las hembras presentaron una longitud total mínima de $8.0 \mathrm{~cm}$, una máxima de $40.4 \mathrm{~cm}$ y promedio de $21.4 \pm 0.49 \mathrm{~cm}$; mientras que los machos presentaron talla mínima de $7.3 \mathrm{~cm}$, máxima de $35.1 \mathrm{~cm}$ y promedio de $22.4 \pm 0.60 \mathrm{~cm}$ (Fig. 1). El ANDEVA mostró que no existió diferencia significativa entre la talla promedio de hembras $\mathrm{y}$ machos $\left(\mathrm{F}_{1,251}=2.964, \mathrm{P}=0.086\right)$.

No fue posible identificar el sexo a 147 individuos; del resto se identificaron 136 hembras (54\%) y 117 machos (46\%), lo que resulta una proporción sexual de 1:0.86 (hembras por machos); esta diferencia no fue estadísticamente significativa $\left(\mathrm{Ji}^{2}=1.03, \mathrm{P}>0.05\right)$ respecto de la proporción teórica esperada 1:1. En el análisis de la proporción de sexos por año (Cuadro 1), solo en 1995 se encontró diferencia estadística significativa. La proporción sexual por clase de longitud (Cuadro 2), no presentó diferencia estadística significativa. Los tres aspectos analizados señalan que la proporción de sexos de $D$. holocanthus está en equilibrio.

Descripción macroscópica de los estadios de desarrollo de las gónadas. En $D$. holocanthus, los ovarios y los testículos se disponen longitudinalmente al cuerpo, forman estructuras pareadas (lóbulos) a manera de sacos y de tamaño aproximadamente similar; los lóbulos se fusionan en la región próxima al 


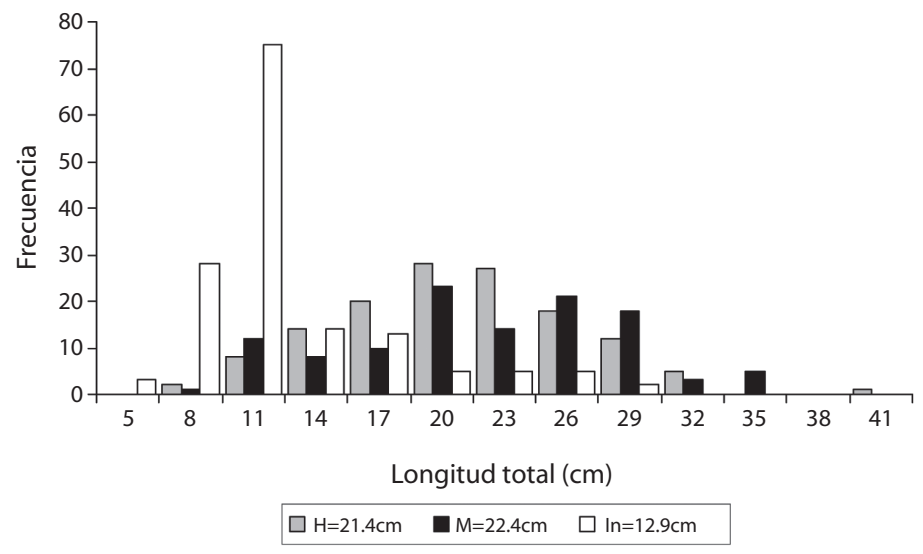

Fig. 1. Distribución de frecuencias de tallas de hembras y machos de $D$. holocanthus en la costa de Jalisco y Colima, México. H=hembras, M=machos y In=indeterminados.

Fig. 1. Length frequency distribution of males and females of D. holocanthus caught in the coast of Jalisco and Colima, Mexico. $\mathrm{H}=$ females, $\mathrm{M}=$ males and In=indeterminate.

\section{CUADRO 1}

Proporción sexual anual de D. holocanthus

TABLE 1

Annual sex ratio of $\mathrm{D}$. holocanthus

$\begin{array}{cccccc}\text { Año } & \text { No } & \begin{array}{c}\text { Proporción } \\ \text { H:M }\end{array} & \begin{array}{c}\text { Valor de } \\ \mathrm{Ji}^{2} \text { calculado }\end{array} & \begin{array}{c}\text { Difiere de } \\ 1: 1\end{array} & \text { P } \\ 95 & 27 & 0.29 & 7.25 & \text { SI } & <0.05 \\ 96 & 68 & 0.70 & 1.77 & \text { NO } & >0.05 \\ 97 & 73 & 0.97 & 0.00 & \text { NO } & >0.05 \\ 98 & 85 & 1.23 & 0.96 & \text { NO } & >0.05\end{array}$

\section{CUADRO 2}

Proporción sexual por clase de talla de D. holocanthus

TABLE 2

Sex ratio by lenght class of $\mathrm{D}$. holocanthus

$\begin{array}{cccccc}\text { Marca de clase }(\mathrm{cm}) & \text { No } & \mathrm{H}: \mathrm{M} & \mathrm{Ji}^{2} & \mathrm{Dif} & \mathrm{P} \\ 7.0-12.9 & 22 & 1.2 & 0.04 & \mathrm{NO} & >0.05 \\ 13.0-15.9 & 23 & 0.53 & 1.56 & \mathrm{NO} & >0.05 \\ 16.0-18.9 & 31 & 0.47 & 3.22 & \mathrm{NO} & >0.05 \\ 19.0-21.9 & 53 & 0.89 & 0.07 & \text { NO } & >0.05 \\ 22.0-24.9 & 42 & 0.5 & 4.02 & \mathrm{SI} & <0.05 \\ 25.0-27.9 & 39 & 1.29 & 0.41 & \text { NO } & >0.05 \\ 28.0-30.9 & 29 & 1.63 & 1.24 & \text { NO } & >0.05 \\ 31.0-42.9 & 14 & 1.33 & 0.07 & \text { NO } & >0.05\end{array}$


orificio urogenital y se encuentran suspendidos en posición anterior a la vejiga natatoria.

Hembras: Inmaduro (I). Los individuos con gónadas inmaduras presentaron una longitud promedio de $15.0 \pm 0.70 \mathrm{~cm}$ y un peso promedio de 192.6 $\pm 23.86 \mathrm{~g}$; las gónadas presentaron peso promedio de $0.6 \pm 0.09 \mathrm{~g}$. En los ovarios se observó una coloración rosa traslúcida y no se observaron huevos a simple vista. Desarrollo (II). Los organismos en este estadio alcanzaron un promedio en la longitud de $20.2 \pm 0.71 \mathrm{~cm}$ y de peso de $414.7 \pm 31.17 \mathrm{~g}$. El peso promedio de los ovarios fue de $7.8 \pm 1.86 \mathrm{~g}$, y coloración rojiza, fue posible distinguir algunos huevecillos a simple vista. Maduro (III). Los ejemplares de este estadio en promedio midieron $23.4 \pm 0.82 \mathrm{~cm}$ y pesaron $612.7 \pm 47.63 \mathrm{~g}$. Las gónadas fueron de mayor tamaño que en los demás estadios, alcanzaron un peso promedio de $40.2 \pm 8.58 \mathrm{~g}$. Los ovarios fueron de color naranja amarillento con vasos sanguíneos superficiales visibles; se observaron los huevos a simple vista. Desovado (IV). Las hembras presentaron una longitud promedio de $25.4 \pm 0.76 \mathrm{~cm}$ y un peso de $640.6 \pm 59.29 \mathrm{~g}$. Las gónadas desovadas alcanzaron un peso promedio de 7.6 $\pm 0.88 \mathrm{~g}$. Los ovarios mostraron una coloración rosa pálido y se observaron restos de huevos. Los ovarios presentaron la superficie rugosa y aspecto de sacos relativamente vacíos.

Machos: Inmaduro (I). La longitud promedio de los machos en este estadio, fue de $15.6 \pm 0.63 \mathrm{~cm}$ y peso promedio de $186.4 \pm 28.81 \mathrm{~g}$. El peso promedio de las gónadas fue de $0.5 \pm 0.08 \mathrm{~g}$. Los testículos fueron de color blanco transparente. Desarrollo (II). Los organismos, mostraron un promedio de talla y peso de $22.8 \pm 0.95 \mathrm{~cm}$ y $496.6 \pm 60.94 \mathrm{~g}$, respectivamente. El peso promedio de las gónadas fue de $2.8 \pm 0.31 \mathrm{~g}$. Los testículos presentaron un color blanco cremoso. Maduro (III). Los organismos de este estadio, mostraron talla promedio de $26.5 \pm 0.71 \mathrm{~cm}$ y peso promedio de $754.2 \pm 44.31 \mathrm{~g}$. Las gónadas alcanzaron peso promedio de $21.6 \pm 2.29 \mathrm{~g}$. Los testículos fueron robustos, firmes y turgentes al tacto, de color blanco nacarado, y presentaron líquido espermático cuando se les aplicó ligera presión. Desovado (IV). La longitud promedio de los organismos fue de $25.9 \pm 0.97 \mathrm{~cm}$ y peso promedio de $718.7 \pm 43.84 \mathrm{~g}$, en las gónadas el peso promedio fue de $8.9 \pm 1.39 \mathrm{~g}$. Los testículos mostraron flacidez, color cremoso pardo y con restos de líquido espermático. En todos los estadios, la capa externa del testículo fue lisa.

\section{Descripción de las fases de ovoci-} tos. Hembras: Fase 1. Ovocitos Cromatina Nucléolo $(\mathrm{CN})$. El área del citoplasma se aprecia reducida comparativamente con el núcleo, dentro del cual, se observaron nucléolos; el diámetro promedio de estos ovocitos fue de $58.6 \pm 0.62 \mu \mathrm{m}$. Fase 2. Ovocitos en Perinucleólo (OP). El diámetro promedio de estos ovocitos fue de $89.3 \pm 1.46 \mu \mathrm{m}$; en la periferia del núcleo se observaron algunos nucléolos; el área del citoplasma se incrementó respecto de los ovocitos de la fase anterior. Fase 3. Ovocitos con Vesículas Vitelinas (VV). En los ovocitos se observó menor cantidad de nucléolos en el núcleo que en aquellos de la fase anterior; se incrementó el área del citoplasma y en el mismo se presentaron vesículas vitelinas, las cuales señalan el inicio de la formación del vitelo primario; estos ovocitos alcanzaron un diámetro promedio de 214.6 $\pm 5.27 \mu \mathrm{m}$. Fase 4. Ovocitos en Vitelogénesis Primaria (VP). Los ovocitos en esta fase presentaron un diámetro promedio de 467.6 $\pm 16.96 \mu \mathrm{m}$; en el núcleo se observaron algunos nucléolos; se incrementó notablemente la superficie del citoplasma, en donde se identificaron vesículas vitelinas y glóbulos de vítelo. Fase 5. Ovocitos en Vitelogénesis Secundaria (VS). En esta fase el diámetro promedio de los ovocitos fue de $687.5 \pm 8.01 \mu \mathrm{m}$; se identificaron algunos nucléolos en el núcleo; nuevamente se observó un incremento sustancial en el área del citoplasma y se observó gran cantidad de glóbulos de vítelo.

Se observó un aumento en el diámetro de los ovocitos según pasan las diferentes fases de desarrollo (progresión de la ovogénesis). En la fase cromatina nucléolo se encontró el 
promedio mínimo $(58.6 \pm 0.62 \mu \mathrm{m})$ y el promedio máximo $(687.5 \pm 8.01 \mu \mathrm{m})$ se presentó en la fase, vitelogénesis secundaria. A medida que avanza el desarrollo de los ovocitos, el diámetro de los mismos se incrementa claramente. El ANDEVA mostró que el incremento en el diámetro difiere de manera significativa entre las distintas fases desarrollo de los ovocitos $\left(\mathrm{F}_{4}\right.$, $1910=3751.127, \mathrm{P}=0.001$ ).

Microscópicamente, se observa que el ovario está envuelto por una capa de tejido muscular o túnica ovárica. Debajo de ésta se forman los sacos ovígeros o lamelas, en donde se desarrollan los ovocitos. Entre los ovocitos se encuentran fibras de tejido conectivo, vasos sanguíneos y epitelio germinal. Dentro del ovario se observaron diferentes fases de maduración de ovocitos, lo cual indica que los ovocitos tienen un patrón de desarrollo asincrónico.

Microscópicamente, en el testículo se observó una membrana que lo rodea, la túnica albugínea. Hacia el centro de la gónada se distinguen septos de tejido intersticial que divide los lóbulos y dentro de éstos se observan los cistos, en su interior se lleva a cabo la espermatogénesis. Cuando se forman los espermatozoides, estos migran hacia el conducto principal del testículo para ser liberados. Por la distribución que presentan los cistos en el interior del testículo, el patrón de organización es de tipo lobular.

Descripción microscópica de los estadios de madurez de $D$. holocanthus. Hembras: Inmaduro o Estadio I. En este estadio se presentaron las tres primeras fases de desarrollo de los ovocitos: ovocitos cromatina nucléolo (CN) con diámetro promedio de $53.3 \pm 1.32 \mu \mathrm{m}$; ovocitos en perinucléolo (OP) teniendo diámetro promedio de $75.5 \pm 1.81 \mu \mathrm{m}$ y ovocitos con vesículas vitelinas (VV) de $166.6 \pm 8.93 \mu \mathrm{m}$ de diámetro (Fig. 2a). En desarrollo o Estadio II. Se observaron las siguientes fases de ovocitos: ovocitos cromatina nucléolo $(\mathrm{CN})$ con diámetro promedio $53.7 \pm 0.94 \mu \mathrm{m}$; ovocitos en perinucléolo $(\mathrm{OP})$ con diámetro promedio de $92.3 \pm 2.25 \mu \mathrm{m}$; ovocitos con vesículas vitelinas (VV) con diámetro de $218 \pm 12.98 \mu \mathrm{m}$, y ovocitos en vitelogénesis primaria (VP) con diámetro promedio de $558.4 \pm 51.74 \mu \mathrm{m}$ (Fig. 2b). Maduro o Estadio III. En este estadio se observaron las cinco fases de desarrollo de ovocitos: ovocitos cromatina nucléolo $(\mathrm{CN})$ con un diámetro promedio de $67.3 \pm 2.27 \mu \mathrm{m}$; ovocitos en perinucléolo (OP) de diámetro promedio $109.8 \pm 5.04 \mu \mathrm{m}$; ovocitos con vesículas vitelinas (VV) de $243.7 \pm 8.42 \mu \mathrm{m}$ de diámetro promedio; ovocitos en vitelogénesis primaria (VP) con diámetro promedio de $484.7 \pm 19.24 \mu \mathrm{m}$ y vitelogénesis secundaria (VS) de $711.8 \pm 7.51 \mu \mathrm{m}$ de diámetro promedio (Fig. 2c). Desovado o Estadio IV. Se presentaron las siguientes fases ovocitos: ovocitos cromatina nucléolo $(\mathrm{CN})$ de $65.3 \pm 0.95 \mu \mathrm{m}$ como diámetro promedio; ovocitos en perinucléolo (OP) con diámetro de $110.2 \pm 3.65 \mu \mathrm{m}$; ovocitos con vesículas vitelinas (VV) de diámetro promedio de $208.5 \pm 6.95 \mu \mathrm{m}$; ovocitos en vitelogénesis primaria (VP) con un diámetro promedio de $309.1 \pm 22.10 \mu \mathrm{m}$ y ovocitos en vitelogénesis secundaria (VS) de $565.5 \pm 21.17 \mu \mathrm{m}$ de diámetro promedio (Fig. 2d).

Machos: Inmaduro o Estadio I. En los testículos de este estadio la túnica albugínea es delgada. El tejido intersticial se encuentra en todo el testículo, pero es más abundante en la parte central. En la parte media del testículo se observan los lóbulos dentro de los cuales se ubican los cistos. (Fig. 3a). En desarrollo o Estadio II. La túnica albugínea es delgada, el tejido intersticial que se observa en el centro del testículo es de menor cantidad que en el estadio anterior debido a la presencia de pequeños grupos de espermatozoides. Se distingue una mayor cantidad de cistos en diferente grado de desarrollo celular (Fig. 3b). Maduro o Estadio III. La túnica albugínea es más gruesa. La cantidad del tejido intersticial es menor, debido a la gran concentración de espermatozoides que se observan. Los lóbulos presentan cistos en diferente grado de desarrollo celular, algunos cistos se encuentran abiertos y vacíos debido a la migración de los espermatozoides hacia el conducto principal (Fig. 3c). Desovado (IV). Túnica albugínea delgada. El tejido intersticial es de mayor cantidad debido a la liberación o 


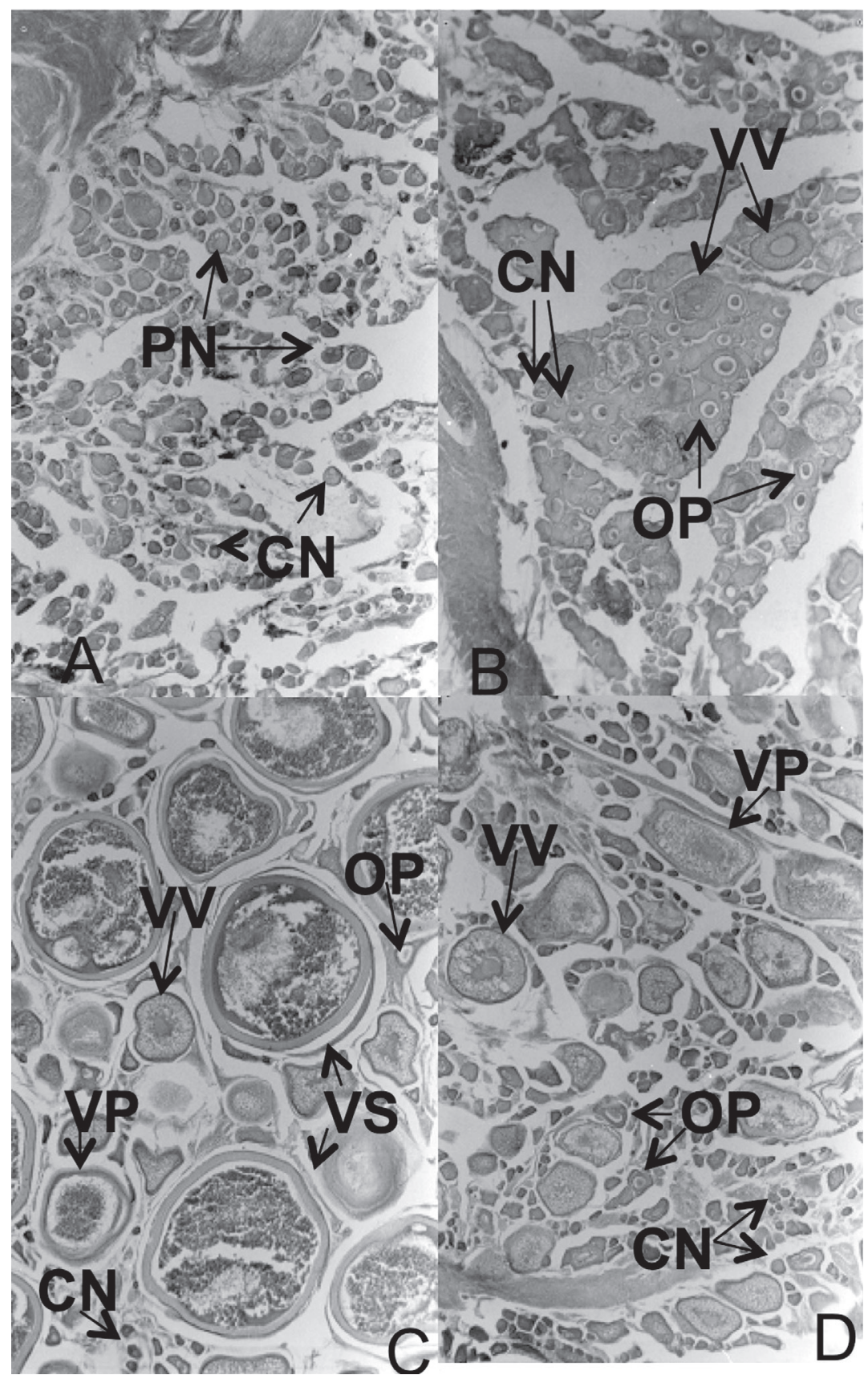

Fig. 2. Cortes de ovarios de D. holocanthus en distintos estadios de madurez mostrando las diferentes fases de desarrollo de los ovocitos. (A) Ovario inmaduro. (B) Ovario en desarrollo. (C) Ovario maduro. (D) Ovario desovado. Ovocitos en cromatina nucléolo $(\mathrm{CN})$, ovocitos en perinucléolo (OP), ovocitos con vesículas vitelinas (VV), ovocitos en vitelogénesis primaria (VP) y ovocitos en vitelogénesis secundaria (VS). Técnica hematoxilina-eosina. 50X.

Fig. 2. Sections of ovarios of $D$. holocanthus in different stages of maturity showing the different phases of development of the oocytes. (A) Inmature ovary. (B) Ovary in development. (C) Mature ovary. (D) Ovary spawn. Chromatin nucleolar oocytes (CN), Perinucleolar oocyte (OP), Yolk vesicles oocytes (VV). Primary yolk oocyte (VP) and Secondary yolk oocyte (VS). Hematoxylin-eosin stain. 50X. 


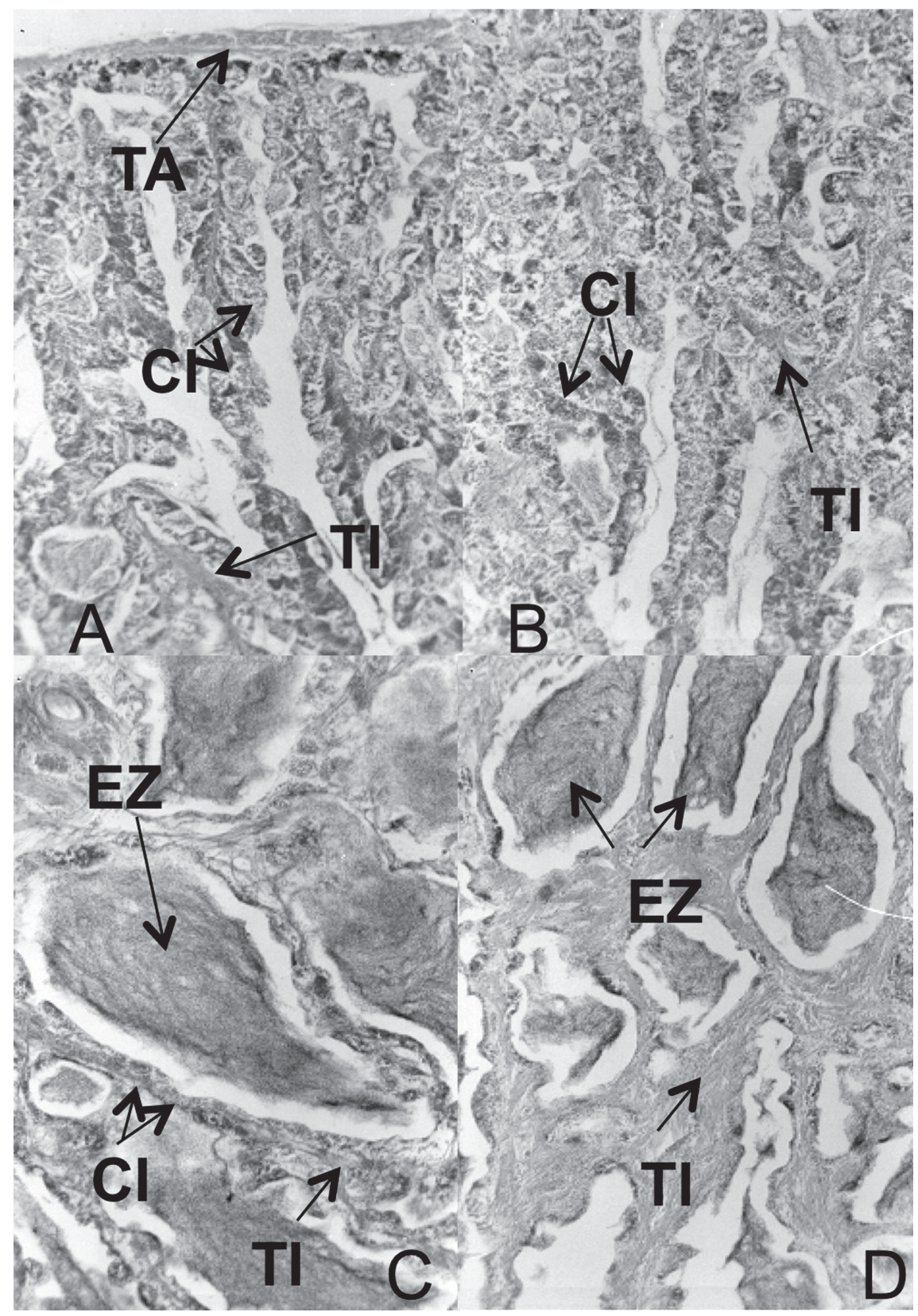

Fig. 3. Corte de testículo de D. holocanthus en distintos estadios de madurez. (A) Testículo inmaduro. (B) Testículo en desarrollo. (C) Testículo maduro. (D) Testículo desovado. Túnica (T), Tejido intersticial (TI), Cistos (CI), grupos de espermatozoides (EZ). Técnica hematoxilina-eosina 100X.

Fig. 3. Sections of testicle of D. holocanthus in different stages of maturity. (A) Inmature testicle. (B) Testicle in development. (C) Mature testicle. (D) Testicle spawn. Tunica (T), Interstitial tissue (TI), Cyst (CI), spermatozoa (EZ). Hematoxylin-eosin satín. 100X. 
desove de los espermatozoides. Se observan pocos cistos con diferente desarrollo celular. Aún se observan algunos espermatozoides agrupados en el conducto principal del testículo (Fig. 3d).

Variación del índice gonadosomático. Se observó que tanto en hembras como en machos, en los primeros meses del año, los valores del IGS se presentaron bajos, en junio ocurrió un incremento en ambos sexos, posteriormente en julio y agosto el IGS disminuyó en hembras y machos. En las hembras, el IGS se incrementa en septiembre y presenta el valor máximo en octubre. En los meses de noviembre y diciembre el IGS disminuyó pero los valores no fueron tan bajos como a inicios del año. Para los machos, el IGS se observó alto de septiembre a diciembre, aunque se presentó una ligera disminución en octubre, pero ésta no es tan baja en comparación como se observó a inicios del año (Fig. 4). Se compararon mediante un ANDEVA los valores mensuales del IGS de ambos sexos y tanto en las hembras $\left(\mathrm{F}_{10,118}=6.324\right.$, $\mathrm{P}=0.001)$, como en los machos $\left(\mathrm{F}_{10,88}=8.243\right.$, $\mathrm{P}=0.001$ ), se presentaron diferencias significativas entre los mes

Variación mensual de los estadios de madurez gonádica: Hembras. Inmaduro (I): El mayor porcentaje de este estadio se presentó en el mes de enero (53.8\%) y el menor porcentaje ocurrió en diciembre $(9.1 \%)$, este estadio se observó en casi todos los meses de muestreo (excepto febrero). Desarrollo (II): A inicio de año se registraron porcentajes importantes de organismos en este estadio, pero no fue hasta junio que alcanzó su mayor porcentaje (80\%) y en noviembre se presentó el menor porcentaje (15.4\%). Maduro (III): Este estadio alcanzó su mayor porcentaje a finales de año a partir del mes de octubre $(66.6 \%)$, le siguió el mes de Noviembre $(61.5 \%)$ y el menor porcentaje se observó en marzo (5\%) (Fig. 5a). Desovado (IV): En el mes de febrero se observó el porcentaje más alto $(100 \%)$ y en el mes de noviembre se presentó el porcentaje más bajo (7.7\%) (Fig. 5a). Las hembras con ovarios maduros se presentaron en junio y de septiembre a diciembre.

Variación mensual de los estadios de madurez gonádica: Machos: Inmaduro (I): El mayor porcentaje de este estadio se presentó en el mes de enero (100\%) y el menor porcentaje se observó en octubre (7.1\%); en febrero se presentó un $50 \%$ de este estadio, y en el resto del año su presencia fue escasa. Desarrollo (II): El mayor porcentaje de este estadio ocurrió en el mes de agosto (66.7\%), el menor se observó en octubre $(7.1 \%)$ y su presencia en el resto del año fue menor. Maduro (III): Este

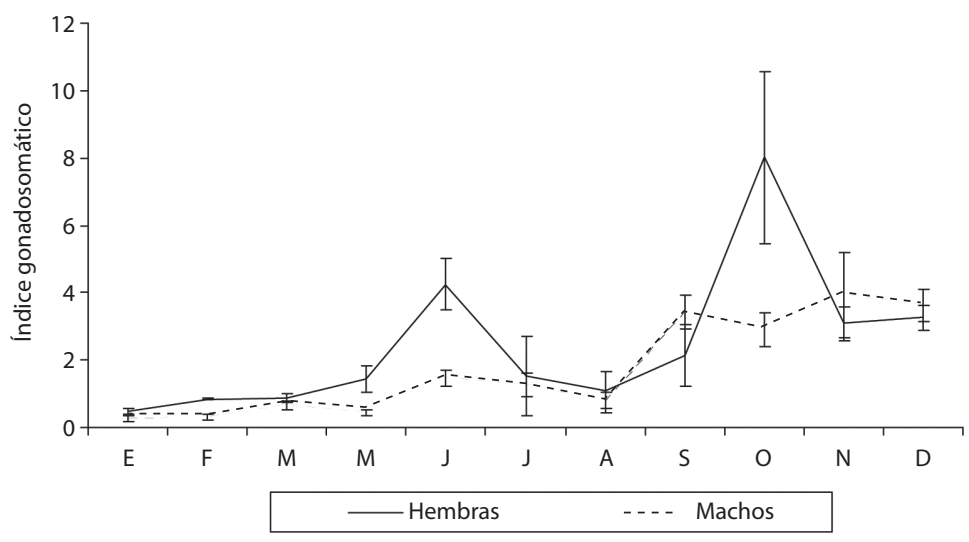

Fig. 4. Variación mensual del índice gonadosomático (media \pm E.S.) de hembras (A) y machos (B) de D. holocanthus. Fig. 4. Monthly variation in the gonadosomatic index (median \pm S.E.) of females (A) and males (B) of D. holocanthus. 

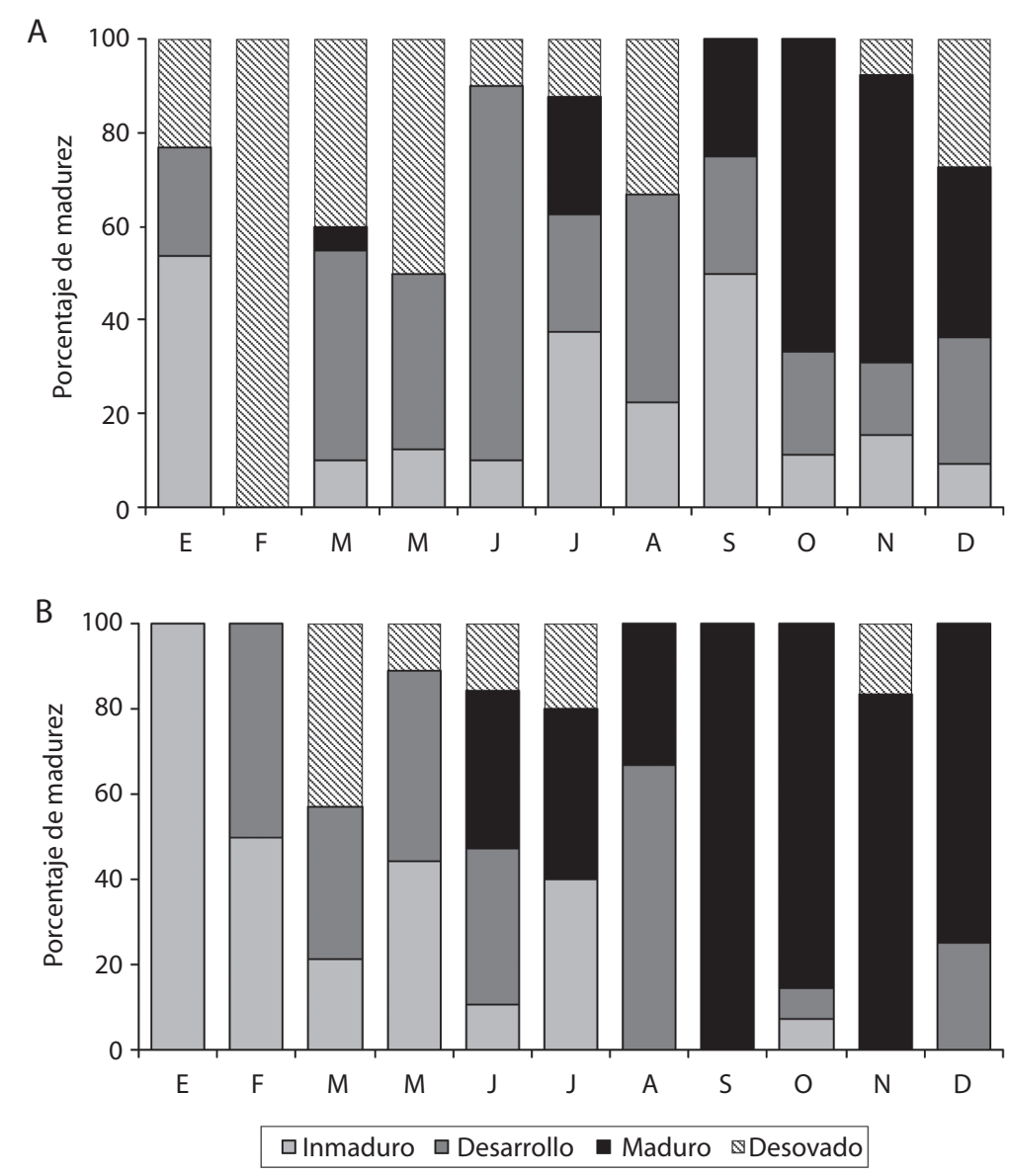

Fig. 5. Porcentaje de los estadios de madurez gonádica de hembras (A) y machos (B) de D. holocanthus en los diferentes meses de muestreo.

Fig. 5. Percentage of the stages of maturity gonadic of female (A) and male (B) of D. holocanthus in the different months of sampling.

estadio alcanzó el $100 \%$ en septiembre, en los siguientes tres meses el porcentaje disminuyó a $85.7 \%, 83.3 \%$ y $75 \%$, respectivamente; en el mes de agosto se registró el porcentaje más bajo (33.3\%). Desovado (IV): Este estadio se presentó en cinco meses, el mayor porcentaje (42.8) se encontró en marzo, mientras que el menor se observó en mayo (11.1\%) (Fig. 5b). Los machos con testículos maduros se presentaron de junio a diciembre.

Diámetro promedio de los ovocitos en diferentes fases: En el Cuadro 3 se presenta el diámetro promedio de los ovocitos para cada uno de los estadios de madurez de las distintas fases de desarrollo encontradas. En general, se aprecia que a medida que se incrementó el desarrollo de la gónada, mayor fue el diámetro de los ovocitos. No se observó una clara diferencia en el diámetro de los ovocitos en las tres primeras fases de desarrollo ( $\mathrm{CN}$, OP y VV) de los cuatro estadios de desarrollo gonadal (inmaduro, en desarrollo, maduro y desovado). Se nota de forma clara, que los ovocitos de las últimas dos fases son mas grandes (VP y VS), los cuales se presentaron en los ovarios 


\section{CUADRO 3}

Diámetro promedio de los ovocitos encontrados en cada uno de los estadios de madurez gonádica de las fases de desarrollo de $\mathrm{D}$. holocanthus

TABLE 3

Diameter average of the oocytes found in each of the stage of gonadic maturity in different stages of development of $\mathrm{D}$. holocanthus

\begin{tabular}{|c|c|c|c|}
\hline Estadio & Fase ovocitos & No. & Media \\
\hline \multirow[t]{3}{*}{ Inmaduro } & Cromatina nucleolo & 195 & $53.3 \pm 1.3$ \\
\hline & Perinucleolo & 166 & $75.5 \pm 1.8$ \\
\hline & Vesículas vitelinas & 40 & $166.6 \pm 8.9$ \\
\hline \multirow[t]{4}{*}{ Desarrollo } & Cromatina nucleolo & 310 & $53.8 \pm 0.9$ \\
\hline & Perinucleolo & 154 & $92.3 \pm 2.3$ \\
\hline & Vesículas vitelinas & 62 & $218.0 \pm 13.0$ \\
\hline & Vitelogénesis primaria & 64 & $432.4 \pm 22.6$ \\
\hline \multirow[t]{5}{*}{ Maduro } & Cromatina nucleolo & 69 & $67.3 \pm 2.3$ \\
\hline & Perinucleolo & 45 & $109.8 \pm 5.0$ \\
\hline & Vesículas vitelinas & 74 & $243.7 \pm 8.4$ \\
\hline & Vitelogénesis primaria & 98 & $484.7 \pm 19.2$ \\
\hline & Vitelogénesis secundaria & 229 & $711.8 \pm 7.5$ \\
\hline \multirow[t]{5}{*}{ Desovado } & Cromatina nucleolo & 289 & $65.4 \pm 0.9$ \\
\hline & Perinucleolo & 47 & $110.2 \pm 3.7$ \\
\hline & Vesículas vitelinas & 50 & $208.5 \pm 6.9$ \\
\hline & Vitelogénesis primaria & 69 & $309.1 \pm 12.6$ \\
\hline & Vitelogénesis secundaria & 123 & $565.5 \pm 12.1$ \\
\hline
\end{tabular}

de mayor desarrollo (en desarrollo, maduro y desovado).

Los diámetros promedio mayores se presentaron en los meses de junio y octubre. El ANDEVA mostró diferencia estadística significativa en el diámetro de los ovocitos en los diferentes meses $\left(\mathrm{F}_{8,1906}=55.534, \mathrm{P}=0.001\right)$. La prueba de comparación múltiple de Tukey, agrupó los diámetros mensuales de los ovocitos en cinco grupos diferentes, entre los cuales, los meses de junio y octubre presentaron los mayores diámetros (Cuadro 4).

Talla promedio de maduración sexual $\left(\mathbf{L}_{\mathbf{5 0}}\right)$ : El $50 \%$ de las hembras de D. holocanthus alcanzó la madurez sexual a los $19.7 \mathrm{~cm}$ de longitud total (Fig. 6a). A su vez se determinó que el 50\% de los machos alcanzó la madurez
CUADRO 4

Comparación múltiple de Tukey del diámetro promedio de los ovocitos encontrados en cada uno de los meses de muestreo

TABLE 4

Multiple comparison of Tukey's test for the average diameter of the oocytes found in each of the months of sampling

\begin{tabular}{lccc}
\multicolumn{1}{c}{ Mes } & No. & Promedio $(\mu \mathrm{m})$ & Grupos \\
Enero & 161 & 79.5 & $\mathrm{X}$ \\
Marzo & 279 & 91.3 & $\mathrm{X}$ \\
Julio & 302 & 146.8 & $\mathrm{X}$ \\
Mayo & 199 & 153.0 & $\mathrm{X}$ \\
Diciembre & 315 & 228.6 & $\mathrm{X}$ \\
Septiembre & 147 & 236.0 & $\mathrm{X}$ \\
Noviembre & 298 & 260.7 & $\mathrm{X}$ \\
Junio & 79 & 347.8 & $\mathrm{X}$ \\
Octubre & 135 & 479.9 & $\mathrm{X}$
\end{tabular}



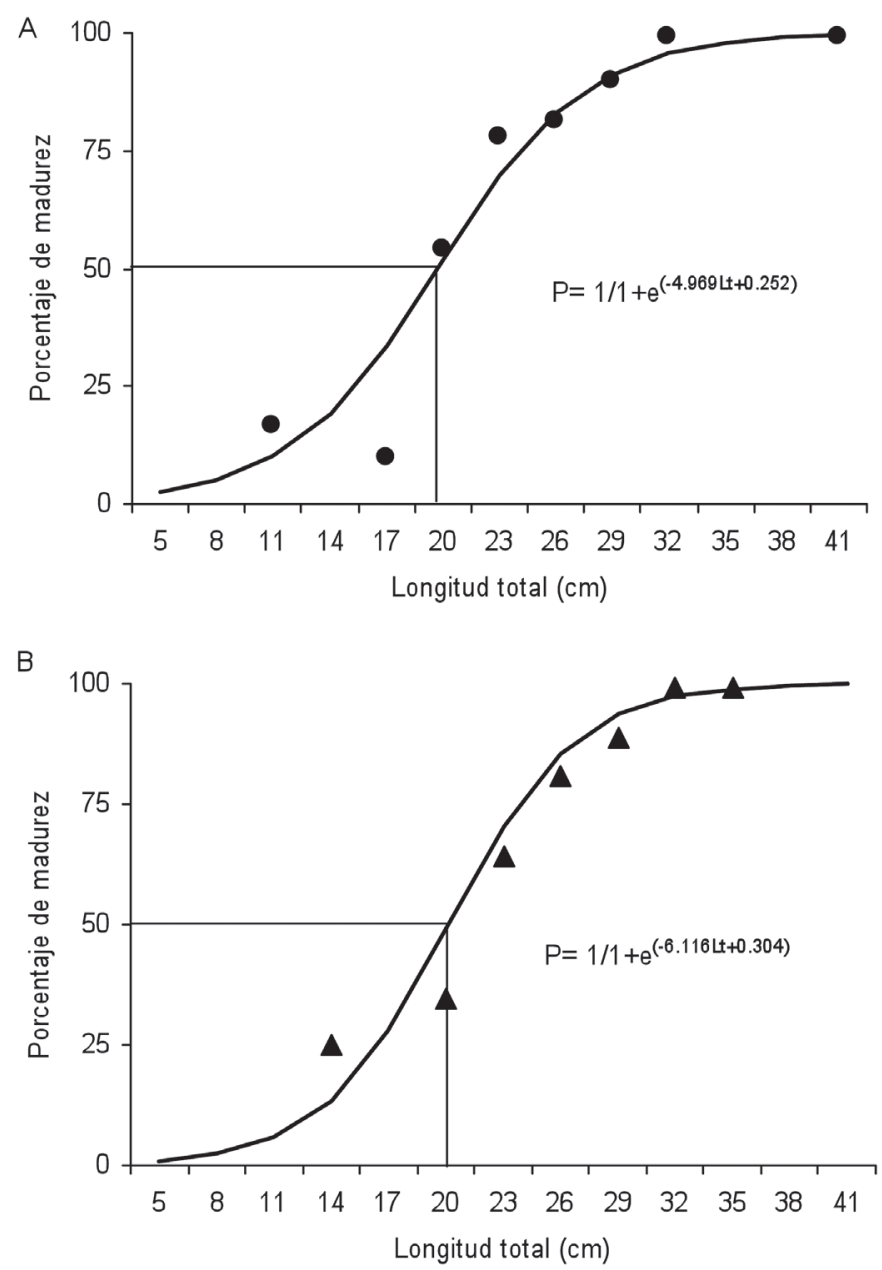

Fig. 6. Porcentaje hembras (A) y machos (B) maduros D. holocanthus recolectados en la costa de Jalisco y Colima, México. Valores ajustados a la ecuación logística.

Fig. 6. Percent of mature female (A) and male (B) D. holocanthus collected in the coast of Jalisco and Colima, Mexico. Data were fitted to the logistic equation.

sexual a los $20.1 \mathrm{~cm}$ de longitud total (Fig. 6b). La hembra y el macho de menor talla que presentaron gónadas en estadio de reproducción registraron tallas de 11.2 y $13.0 \mathrm{~cm}$ de longitud total, respectivamente.

\section{DISCUSIÓN}

D. holocanthus es una especie que no tiene gran importancia en el comercio y consumo humano directo, sin embargo, posee cierto valor económico en algunos estados de México, ya que de los ejemplares preparados se venden como artesanía (Holguín-Quiñones 1976). Lo anterior también ocurre en algunas poblaciones de la región costera de Jalisco y Colima (observación personal). Además, las etapas juveniles de esta especie se comercializan en acuarios como especies de ornato (Patton 2009). En China esta especie se emplea en la medicina tradicional (Tang 1987). 
La longitud máxima encontrada en este trabajo $(40.3 \mathrm{~cm})$ resultó superior a la encontrada por otros autores. Para esta misma especie, Allen \& Robertson (1994), en el Pacífico oriental tropical, registraron una talla máxima de $29.0 \mathrm{~cm}$. Raymundo-Huizar \& Chiapa-Carrara (2000) reportan una talla de $30.5 \mathrm{~cm}$. Respecto a la talla mínima, en este estudio se registraron tallas de $5.0 \mathrm{~cm}$, similar a la encontrada por Raymundo-Huizar \& Chiapa-Carrara (2000).

Un elemento importante en la reproducción de los peces es la proporción de hembras y machos que integran una población. Sólo para el año de 1995 se encontró diferencia en la proporción sexual. Una explicación plausible, es que los datos de este año, sólo fueron obtenidos en un mes (diciembre). Por este motivo, se considera que la población de D. holocanthus en la región de estudio está en equilibrio sexual. No se localizaron referencias de trabajos sobre este aspecto en esta especie. En general, para muchas especies de peces, una proporción sexual diferente al equilibrio puede deberse a que en algunas situaciones las hembras tienen mayor supervivencia que los machos o diferencias en la distribución y el hábitat que prefiere cada sexo (Santamaría-Miranda 1998).

Diferentes autores han empleado la variación temporal del IGS para determinar el periodo de reproducción en muchos peces (Lucano-Ramírez 1998, Palomera-Sánchez 2004). Everson et al. (1989) y Gillanders et al. (1999), ubican el periodo de reproducción mediante el diámetro de los ovocitos. En $D$. holocanthus los máximos valores del diámetro de los ovocitos ocurren en los mismos meses con picos en el IGS y con el mayor porcentaje de individuos con gónadas maduras. Estas coincidencias refuerzan la idea de que la actividad reproductiva de esta especie tiene mayor intensidad en junio y en el periodo de septiembre-diciembre. Para D. holocanthus, Fujita et al. (1997) en Okinawa, Japón, registraron un pico en el IGS de hembras, el cual ocurrió en el mes de mayo. Entre último trabajo y el presente estudio existe una similitud, al presentar los valores máximos del IGS en meses contiguos (mayo y junio). También se observa una diferencia, en el presente estudio se registró un segundo pico en el IGS, el cual tiene mayor duración. Estas diferencias pueden deberse a la distinta localización geográfica de cada estudio, que conlleva condiciones ambientales también diferentes. Se conoce que especies de peces que viven en climas tropicales y subtropicales, pueden presentar varios periodos reproductivos en comparación con especies de clima templado (Lagler et al. 1984).

El patrón de desarrollo de los ovocitos de D. holocanthus es de tipo asincrónico, el cual consiste en presentar en el ovario ovocitos en varias etapas de desarrollo. Los peces pertenecientes a este grupo generalmente tienen un largo periodo de desove (Peralta-Carranco 1991). Este mismo patrón de desarrollo de ovocitos, lo presentan muchas especies de peces como es el caso del huachinango $L$. peru (Reyna-Trujillo 1983, Lucano-Ramírez 1998, Santamaría-Miranda 1998), PalomeraSánchez (2004) en su trabajo con Scomberomorus Sierra, observó el mismo desarrollo y de la misma manera lo observaron Arcos-Huitrón \& Torres-Villegas (1990) en Baja California Sur en la macarela Scomber japonicus. En aguas continentales como en el Lago de Patzcuaro, Michoacán, Peralta-Carranco (1991) también encontró este tipo de desarrollo, en el Chirostoma estor copándaro.

Diversos autores han descrito un número diferente de fases en el desarrollo de los ovocitos. Yamamoto \& Yamazaki (1961) describen diez fases, Cerisola (1984) reseña ocho fases, Lucano-Ramírez et al. (2001c) definen siete fases y Palomera-Sánchez (2004) registró cinco fases de desarrollo. En el presente trabajo se observaron cinco fases, al igual que PalomeraSánchez (2004). Muchas de las características del desarrollo del ovocito en D. holocanthus, son muy similares a las que describen los autores arriba mencionados. La diferencia entre el número de fases encontradas en los diferentes estudios puede deberse a un efecto del tamaño, la intensidad y las condiciones del muestreo o debido a que algunas fases ocurren de manera muy acelerada y su observación se hace difícil. 
En el proceso de desarrollo normal del ovario, el aumento de biomasa corresponde a un mayor tamaño de los ovocitos de las distintas fases que lo integran. Para $D$. holocanthus se observó un aumento del promedio del diámetro de los ovocitos en los estadios III (maduro) y IV (desovado). Conforme mayor es el tamaño de la gónada, mayor será el tamaño de los ovocitos en distintas fases. Lo anterior también se presentó en el huachinango, Lutjanus peru (Lucano-Ramírez 1998) y en la sierra, $S$. sierra (Palomera-Sánchez 2004).

El desarrollo testicular puede variar de una especie a otra. Palacios-Ceballos (1995), menciona que existen básicamente dos tipos de desarrollo testicular: el lobular y el tubular. Este autor encontró que en la tilapia del Nilo, Oreochromis niloticus, el testículo presentó un desarrollo de tipo lobular (que es típico en peces teleósteos). Este mismo tipo lo presentó L. peru en la costa sur de Jalisco (LucanoRamírez 1998) y es el que muestra D. holocanthus. En este tipo de desarrollo, cada lóbulo del testículo contiene numerosos cistos. Dentro de cada cisto los grupos de células germinales se dividen sincrónicamente y todas se encuentran en la misma fase de desarrollo en un tiempo dado. Por otra parte, dentro de los lóbulos el proceso de espermatogénesis no es sincrónico, lo que quiere decir que se pueden encontrar cistos en diferentes fases de desarrollo; posteriormente, durante la espermatogénesis éstos se rompen para liberar los espermatozoides (Lucano-Ramírez, 2001b).

Los resultados obtenidos en este trabajo sugieren que los dos sexos maduran a una talla muy similar.

\section{AGRADECIMIENTOS}

A los investigadores, estudiantes del Departamento de Estudios para el Desarrollo Sustentable de Zonas Costera, así como a la tripulación del BIP V que participaron en los muestreos. Este trabajo se realizó bajo el permiso de Pesca de Fomento de la SEMARNAP No. 150995-214-03. La investigación fue financiada por la Universidad de Guadalajara y el CONACYT

\section{RESUMEN}

Diodon holocanthus es una especie con cierta importancia comercial y ecológica en la comunidad de peces que forma la fauna de acompañamiento del camarón en la plataforma continental del Pacífico central Mexicano, y de la cual no se conocen aspectos reproductivos. Por lo cual se obtuvieron el periodo de reproducción, la descripción macro y microscópica de las gónadas, además de variables poblacionales como: distribución de tallas, proporción sexual y talla de madurez. En total se capturaron alrededor de 400 organismos, los cuales presentaron una talla mínima de $5.0 \mathrm{~cm}$, máxima de $40.3 \mathrm{~cm}$ y promedio de $18.4 \mathrm{~cm}$, y fueron capturados en la plataforma continental en el Pacífico central Mexicano, desde diciembre de 1995 a diciembre de 1998. La proporción sexual fue de 1:0.86 hembras por machos $(\mathrm{n}=253)$. La talla a la cual el $50 \%$ de individuos presentó gónadas maduras fue $19.7 \mathrm{~cm}$ en las hembras y $20.1 \mathrm{~cm}$ en los machos. Los organismos con las menores tallas que presentaron gónadas en fase de maduración midieron $12.2 \mathrm{~cm}$ (hembras) y $13 \mathrm{~cm}$ (machos). En ambos sexos el desarrollo de la gónada se determinó con base en una escala de maduración de cuatro estadios. En el proceso de maduración de los ovocitos se identificaron cinco fases de desarrollo. El patrón de desarrollo de los ovocitos es de tipo asincrónico, lo que significa que la especie se puede reproducir varias veces al año. El desarrollo del testículo es de tipo lobular como en la mayoría de los peces teleósteos. El índice gonadosomático sugiere que el periodo de reproducción se concentra en los meses de junio, y de septiembre a diciembre.

Palabras clave: reproducción, Diodontidae, pez globo, maduración gonadal, proporción sexual.

\section{REFERENCIAS}

Acal, D.E. \& A. Arias. 1990. Evaluación de los recursos demerso-pelágicos vulnerables a redes de arrastre de fondos en el Sur del Pacífico de México. Cien. Mar. 16: 93-129.

Aguilar-Palomino B., J. Mariscal-Romero, G. GonzálezSansón \& L.E. Rodríguez-Ibarra. 1996. Ictiofauna demersal de fondos blandos de la plataforma continental de Jalisco y Colima, México, en la primavera de 1995. Cien. Mar. 22: 469-481.

Aguilar-Palomino, B., C. Pérez-Reyes, F. Galván-Magaña \& L.A. Abítia-Cárdenas. 2001. Ictiofauna de la Bahía de Navidad, Jalisco, México. Rev. Biol. Trop. 49: 173-190. 
Allen, G.R. \& R. Robertson. 1994. Fishes of the Tropical Eastern Pacific. University of Hawaii, Hawaii, EEUU.

Arcos-Huitrón, N.E \& J.R. Torres-Villegas. 1990. Ciclo reproductivo de la macarela del Pacífico Scomber japonicus Houttyn (Pisces: Scombridae) en Bahía Magdalena, Baja California Sur, México. Invest. Mar. CICIMAR 5: 37-45.

Bussing, W.A. \& M.I. López 1994. Peces demersales y pelágicos costeros del Pacífico de Centro América meridional: Guía ilustrada.

Chen, C.Y. \& H.N. Chou. 1998. Detection of tetrodotoxin by high performance liquid chromatography in linedmoon shell and puffer fish. Acta Zool. Taiw. 9: 41-48.

Cerisola, H. 1984. Cambios estructurales del folículo ovárico durante su maduración en el pejesapo Scyases sanguineus, Muller y Troschel 1843. (Gobiescocidae teleostei). Rev. Biol. Mar. 20: 1-21.

Echeverria, W.T. 1987. Thirty-four species of California rockfishes: maturity and seasonality of reproduction. Fish. Bull. 85: 229-250.

Everson, A.R., H.A. Williams \& B.M. Ito. 1989. Maturation and reproduction in two hawaiian eteline snappers, Uku, Aprion virescens, and Onaga, Etelis coruscans. Fish. Bull. 87: 877-888.

Fujita, T., W. Hamaura, A. Takemura \& K. Takano. 1997. Histological observations of annual reproductive cycle and tidal spawning rhythm in the female porcupine fish Diodon holocanthus, in Okinawa, southern Japan. Fish. Sci. 65: 715-720.

Galván-Magaña, F., L.A. Arbitia-Cárdenas, J. RodríguezRomero, H. Pérez-España \& H. Chávez-Ramos. 1996. Lista sistemática de los peces de Isla Cerralvo Baja California Sur, México. Cien. Mar. 22: 295-311.

Gillanders, B.M., D.J. Ferrell \& N.L. Andrew. 1999. Size at maturity and seasonal changes in gonad activity of yellowtail kingfish (Seriola lalandi, Carangidae) in New South Wales, Australia. New Zeal. Journ. Mar. Fresh. Res. 33: 457-468.

González-Sansón, G., B. Aguilar-Palomino, J. ArciniegaFlores, R. García de Quevedo-Machain, E. GodínezDomínguez, V. Landa-Jaime, J. Mariscal-Romero, J.E. Michel-Morfín \& M. Saucedo-Lozano. 1997. Variación espacial de la abundancia de la fauna de fondos blandos en la plataforma continental de Jalisco y Colima, México (Primavera, 1995). Cien. Mar. 23: $93-110$
Holden, M.J. \& D.F.S. Raitt. 1975. Manual of fisheries science. Part 2. Methods of resource investigations and their application. FAO Fish. Pap. 115: 1-214.

Holguín-Quiñónez, O.E. 1976. Catálogo de especies marinas de importancia comercial de Baja California Sur. Inst. Nac. Pesca, Subsecretaría de pesca. La Paz, B.C.S., México.

Hyder, M. 1969. Histological studies on the testis of tilapia leucosticta and other species of the genus Tilapia (Pises: Teleostei). Trans. Amer. Microsc. Soc. 88: 211-231.

Lagler, K.F., J.E. Bardach, R.R. Millar \& D.M. Passino. 1984. Ictiología. AGT Editor, México D.F., México.

Lucano-Ramírez, G. 1998. Ciclo Reproductivo de Lutjanus peru (Nichols y Murphy, 1922) (Pisces: Lutjanidae) en la costa sur de Jalisco. Tesis de Maestría, Universidad Autónoma de México, México D. F., México.

Lucano-Ramírez, G., S. Ruiz-Ramírez, B. Aguilar-Palomino \& J.A. Rojo-Vázquez. 2001a. Listado de las especies de peces de la región costera de Jalisco y Colima. Ciencia y Mar. V: 13-20.

Lucano-Ramírez, G., M. Villagrán-Santa Cruz \& S. RuizRamírez. 2001b. Cambios estructurales en las gónadas de Lutjanus peru en la costa sur de Jalisco, México. Bol. Centr. Invest. Biol. 35: 223-372.

Lucano-Ramírez, G., M. Villagrán-Santa Cruz, S. RuizRamírez \& T. López-Murillo. 2001c. Histología de los ovocitos de Lutjanus peru (Nichols and Murphy, 1922) (Pisces: Lutjanidae). Cien. Mar. 27: 335-349.

Martínez, A.J. 1988. Guía ilustrada para la identificación de peces, UNAM, ENEP Iztacala, Mexico.

Nagelkerken, I. \& M. Dorenbosch. 2000. Day-night shifts of fishes between shallow-water biotopes of a Caribbean bay, with emphasis on the nocturnal feeding of Haemulidae and Lutjanidae. Mar. Ecol. Prog. Ser. 194: 55-64.

Palacios-Ceballos, R. 1995. Estudio del patrón espermatogenico de la tilapia del nilo Oreochromis niloticus (PISCIES: CICHLIDAE). Tesis de Licenciatura, Universidad Autónoma de México, México D.F., México.

Palomera-Sánchez, F.I. 2004. Características reproductivas de Scomberomorus sierra Jordan y Starks 1895 (PISCES: SCOMBRIDAE) en Bahía Navidad, Jalisco, México. Tesis de Licenciatura, Universidad de G. Autlán de Navarro, Jalisco, México. 
Peralta-Carranco, C. 1991. Ciclo gonádico a nivel histológico en hembras de Chirostoma estor copándaro (Pescado blanco) en el Lago de Pátzcuaro Michoacán. Tesis de Licenciatura, Universidad Autónoma México, México, D.F., México.

Patton, C. 2009. Florida Museum of Natural History (FLMNH), "Balloonfish". (Downloaded: October 18, 2009, http://www.flmnh.ufl.edu/fish/Gallery/Descript/Balloon/Balloon.htm).

Ramírez-Hernández, E. \& G. Carrillo-González. 1961. Investigaciones ictiológicas en la costa de Chiapas. Listado de peces colectados en las capturas camaroneras (agosto-septiembre y abril-mayo y junio 1960). Inst. Nac. Invest. Biol. Pesq., Secretaría de Industria y Comercio, Dirección General de Pesca e Industrias Conexas, México.

Ramírez-Hernández, E. \& J. Páez-Barrera. 1964. Investigaciones ictiológicas en las costas de Guerrero I. Lista de peces marinos de Guerrero colectados en el periodo 1961-1965. An. Inst. Nac. Invest. Biol. Pesq. 1: 329-360.

Randall, J.E., 1967. Food habits of reef fishes of the West Indies. Stud. Trop. Oceanog. 5: 665-847.

Raymundo-Huizar, A.R. \& X. Chiapa-Carrara. 2000. Hábitos alimentarios de Diodon histrix y Diodon holocanthus en las costas de Jalisco y Colima, México. Bol. Cent. Invest. Biol. 34: 181-210.

Reyna-Trujillo, M.M. 1993. Desarrollo gonádico y época de desove del huachinango (Lutjanus peru) Nichols y Murphy 1922 (Pisces:Lutjanidae) en la Bahía de la
Paz, B.C.S. México. Tesis de Licenciatura, Universidad de G. Guadalajara, Jalisco, México.

Rodríguez-Gutiérrez, M. 1992. Técnicas de evaluación cuantitativas de la madurez gonádica en peces. AGT Editor, México.

Rojo-Vazquez, J.A., B. Aguilar-Palomino, V.H. GalvánPiña, E. Godínez-Domínguez, S. Hernández-Vázquez, S. Ruíz-Ramírez \& G. Lucano Ramírez. 2001. Ictiofauna de la pesquería ribereña en Bahia de Navidad, Jalisco, México, asociada al evento El Niño 1997-1998. Rev. Biol. Trop. 49: 915-929.

Santamaría-Miranda, A. 1998. Hábitos alimenticios y ciclo reproductivo del huachinango Lutjanus peru, (Nichols y Murphy, 1922) en Guerrero, México. Tesis de Maestría, CICIMAR-IPN, La Paz B.C.S., México.

Santos-Martínez, A. 1989. Estudio biológico y ecológico de la ictiofauna de la Ciénaga Grande de Santa Marta, Caribe Colombiano. Tesis de Maestría, Universidad Nacional de Colombia, Bogotá, Colombia.

Tang, W.C. 1987. Chinese medicinal materials from the sea. Abst. Chin. Med. 1: 571-600.

Van Der Heiden, A.M. \& L.T. Findley. 1988. Lista de peces marinos del sur de Sinaloa, México. An. Cien. Mar Limnol. UNAM 15: 209-224.

Yamamoto, K. \& F. Yamazaki. 1961. Rhythm of Development in the Oocyte of the Gold-Fish (Carassius auratus). Bull. Fac. Fish., Hokkaido University 12: 93-114.

Zar, J.H. 1996. Bioestatistical analysis. Prentice Hall, Nueva Jersey, EEUU. 